\title{
Effect of rice AUXIN BINDING PROTEIN57 (OsABP57) overexpression in response to flooding
}

\begin{abstract}
This study reports the effects of Oryza sativa AUXIN BINDING PROTEIN57 (OsABP57) overexpression towards flooding in rice. OsABP57 was previously reported to activate plasma membrane H+-ATPase. Earlier studies address the ability of transgenic OsABP57 overexpression in enduring drought and salinity stresses but none on the flooding. In this study, complete submergence analysis was carried out and several morphophysiological parameters were analyzed such as plant height, root architecture and relative chlorophyll content. Results showed that there are no differences between OsABP57 overexpression rice compared to MR219 control rice in terms of chlorophyll content and plant height after 1-3 weeks of flooding treatments. Root analysis, however, found that transgenic rice OsABP57 produced more adventitious roots compared to MR219 rice under normal condition, which may be due to the role of the gene that encodes for auxin binding protein. The semi-quantitative polymerase chain reaction (PCR) on Oryza sativa pyruvate decarboxylase (OsPDC) gene after two weeks of flooding treatment showed an increase of expression in OsABP57 transgenic compared to MR219. Overall, the overexpression of OsABP57 did not show any significant difference in terms of morphophysiological analysis between the transgenic line and MR219, yet, there is an increase of OsPDC gene in the transgenic background which may need further experimental analysis in the future to map the network between auxin and hypoxia core genes.
\end{abstract}

Keyword: Oryza sativa; Flooding; OsABP57; MR219; Hypoxia 